\title{
STEERING AND LOCKING OF DISCRETE SOLITONS IN OPTICAL WAVEGUIDE ARRAYS
}

\author{
U. Peschel, F. Lederer \\ Department of Physics and Astronomy, Friedrich-Schiller University Jena \\ Max-Wien-Pl. 1, 07743 Jena, Germany \\ R. Morandotti, J.S. Aitchison \\ Department of Electronics and Electrical Engineering, University of Glasgow \\ Glasgow, Scotland, UK \\ H. Eisenberg and Y. Silberberg \\ Department of Physics of Complex Systems, The Weizmann Institute of Science \\ Rehovot, Israel
}

By operating an AlGaAs waveguide array at the half band gap, we experimentally demonstrate basic features of discrete systems, which do not appear in the corresponding continuous counterpart - the slab waveguide. Under selected initial conditions, we observed nonlinearly induced locking of an initially moving soliton to the input waveguide. If the power is even higher, originally locked beams start to move again, but in a direction opposite to the initial tilt.

PACS numbers: 42.65.Tg, 42.65.Wi, 42.82.Et

\section{Introduction}

Spatial solitons can be easily generated in planar waveguides, where the slab confines one of the dimensions [1]. They originate from a balance between the material non-linearity and diffraction. By imposing a phase gradient on the generating beam they can be steered into a desired direction, a property that can be used for e.g. routing applications in optical fiber networks. The existence of spatial solitons has also been predicted for an infinite array of identical, weakly coupled waveguides $[2,3]$. In such a structure, when low intensity light is injected into either one or just a few waveguides, it will couple and spread to an increasing number of guides as it propagates, thereby broadening its spatial distribution. This widening of the field distribution is analogous to diffraction in continuous media. By injecting a strong optical field, it is possible to modify the refractive index of 
the excited waveguides through the optical nonlinearity of the material and to decouple them from the rest of the array. In ideal conditions, particular high intensity beams propagate while keeping a fixed spatial profile localized in a limited portion of the array, as spatial solitons do in a slab waveguide [4]. Due to this similarity, these quasistationary excitations are called discrete spatial solitons. Their behavior is rather general. Many natural systems consist of simple elements, which are periodically ordered and mutually interacting. Localized modes in molecular chains [5], in electrical lattices [6], in Josephson junction ladders [7] or in a coupled array of mechanical pendula [8] as well as polarons in ionic crystals [9] are just a few examples of energy localization in discrete systems. It turns out that in particular the dynamical properties of these solitary waves or discrete breathers [10], which we are about to investigate in this paper, differ from the continuous case considerably.

The paper is structured as follows. First we explain the experimental setup, the methods of measurement used and the way we used to model the experiment. The discussion of the experimental results focuses on the power induced locking of solitons generated by a tilted beam. A short summary concludes the paper.

\section{Experimental realization and modeling}

The sample employed for the measures (see Fig. 1) was an array of 41 waveguides etched $0.9 \mu \mathrm{m}$ in the cladding of an AlGaAs slab, which was composed of a core of $\mathrm{Al}_{0.18} \mathrm{Ga}_{0.82} \mathrm{As}, 1.5 \mu \mathrm{m}$ thick, sandwiched between two cladding layers composed of $\mathrm{Al}_{0.24} \mathrm{Ga}_{0.76} \mathrm{As}$. The upper layer was $1.5 \mu \mathrm{m}$ and the lower was $4.0 \mu \mathrm{m}$ thick. These waveguide dimensions were chosen to guarantee single mode operation for a fixed polarization direction. The guides, $4 \mu \mathrm{m}$ wide, had a distance center-to-center equal to $9 \mu \mathrm{m}$. The sample was $6 \mathrm{~mm}$ long. The experimental setup is represented in Fig. 1, which is completed by a micrograph of the array. To carry out the experiments reported in this paper, we used a Spectra Physics Beam Lock-Tsunami-Opal system. The emission wavelength of the parametric oscillator was $1530 \mathrm{~nm}$, delivered under the form of a train of pulses with a length of about $150 \mathrm{fs}$ and a repetition rate of $80 \mathrm{MHz}$. This wavelength, below half the band gap of the material, was chosen in order to minimize the detrimental effects of non-linear absorption. To excite the modes in the sample we used elliptically shaped TE polarized beams with a fixed height of $3 \mu \mathrm{m}$, but with three different widths equal to $9 \mu \mathrm{m}, 13 \mu \mathrm{m}$, and $20 \mu \mathrm{m}$ respectively.

Note that only the field at the output facet could be recorded with an infrared camera. Any insight into the field evolution in the array can only be obtained by means of numerical simulations. To model the field evolution in the array a coupled mode theory was applied. Here we assume that the mode profile in the individual guides remains unchanged, where only the amplitudes $a_{n}$ evolve with respect to time $t$ and propagation direction $z$ as

$$
\left(\mathrm{i} \frac{\partial}{\partial z}-\frac{D}{2} \frac{\partial^{2}}{\partial t^{2}}+\mathrm{i} \frac{\alpha_{1}}{2}+\gamma\left|a_{n}\right|^{2}+\mathrm{i} \alpha_{3}\left|a_{n}\right|^{4}\right) a_{n}+C\left(a_{n+1}+a_{n-1}\right)=0,
$$

where the quantities involved are the chromatic dispersion $D=1350 \mathrm{ps}^{2} / \mathrm{km}$, the linear losses $\alpha_{1}=0.9 \mathrm{~cm}^{-1}$, the Kerr nonlinearity $\gamma=3.6 \mathrm{~m}^{-1} \mathrm{~W}^{-1}$, the 


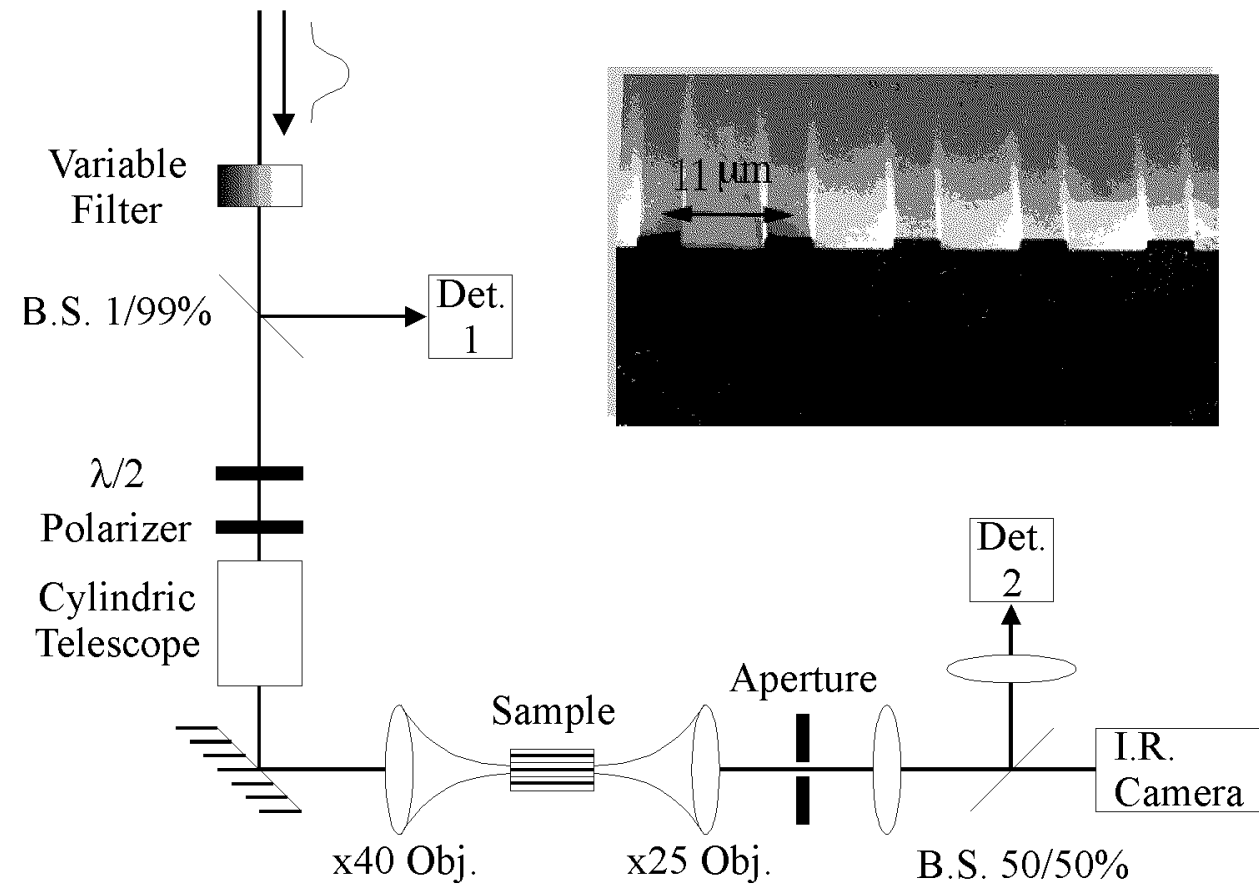

Fig. 1. Experimental setup and a viewgraph of a sample (B.S. = beam splitter).

three photon absorption coefficient $\alpha_{3}=10^{-4} \mathrm{~m}^{-1} \mathrm{~W}^{-1}$ and the coupling term $C=0.82 \mathrm{~mm}^{-1}$. All these values, except the linear damping, were derived from a calculation of the mode profiles and by using material constants published in the literature. The linear damping, which is mainly due to scattering losses on imperfections, was determined by evaluating the Fabry-Perot fringes shown by the sample operated in transmission.

Only if quantitative agreement was required we used Eq. (1) including losses and transient effects. To identify typical effects of discreteness most of the simulations were based on a more simplified version of Eq. (1). It turned out that most of the phenomena observed can already be understood if the time dependence of the field amplitudes is neglected. Note that if additionally all dissipation is neglected, Eq. (1) transforms into the well known so-called discrete nonlinear Schrödinger equation (DNLS).

\section{Experimental results and interpretations}

\subsection{Soliton formation and beam break-up}

Although it is not the main subject of this paper, we start with a brief discussion of soliton formation in waveguide arrays. The evolution of untilted beams, which are centered on a guide, is rather similar to that in the continuous case. 

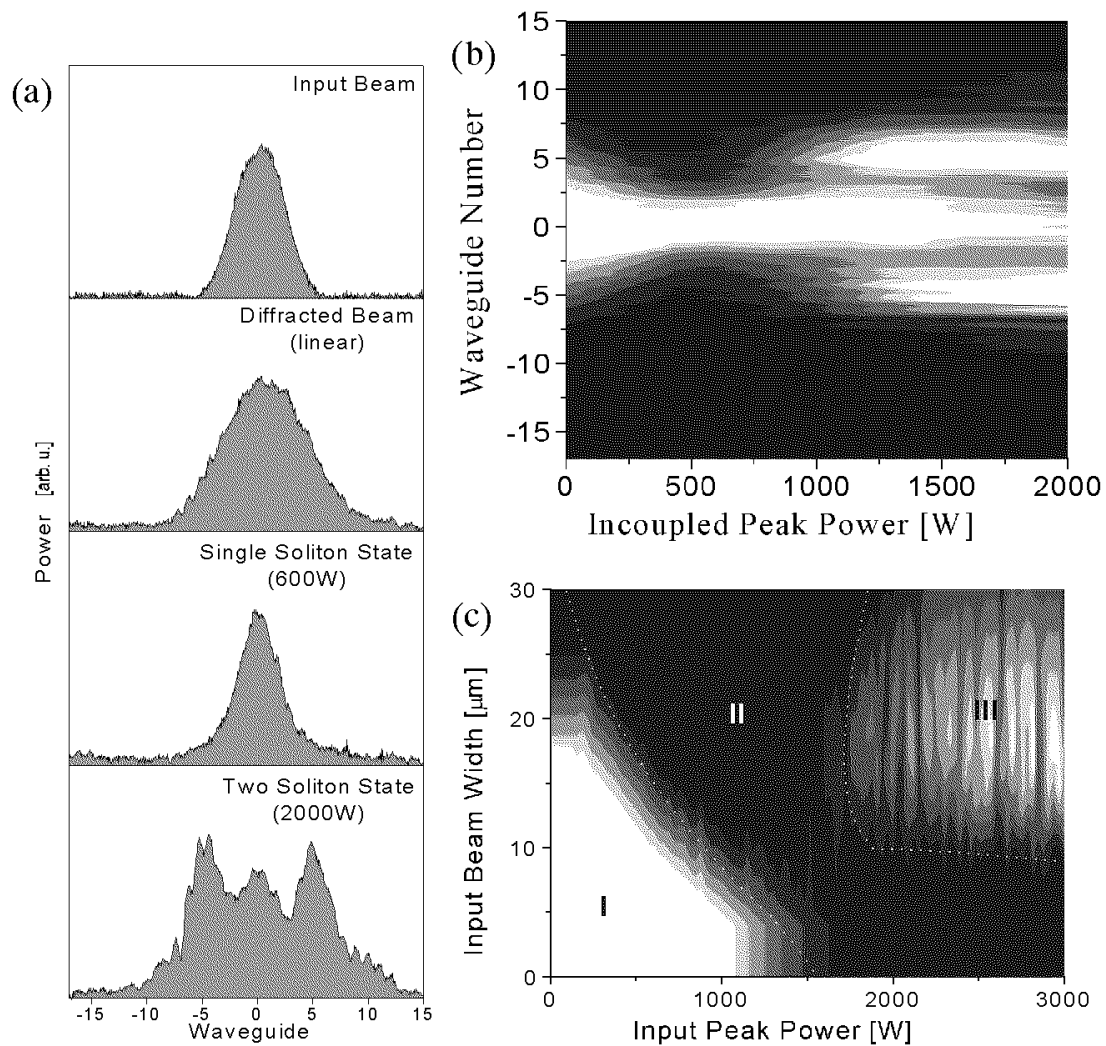

Fig. 2. Soliton formation and beam break-up: (a) Experimentally recorded field structures for a wide input beam (beam width $20 \mu \mathrm{m}$ ) and for different power levels; (b) experimentally recorded output field profile for varying input power levels; (c) simulation of the width of the output field distribution on the basis of the complete model (see Eq. (3)) (white: wide output beam, black: narrow output beam). In dependence from the beam size and the input power three different scenarios can be distinguished: I: linear diffractive spreading, II: formation of a discrete soliton, III: soliton break-up.

Three different scenarios can be distinguished, corresponding to three different areas in a parameter space spread by the incoupled power and the beam width (see Fig. 2c). In the linear case the beam diffracts. The narrower the exciting beam the more power is required to balance the spreading and to form a discrete soliton. If the beam is wide enough and therefore close to the continuous limit the beam finally splits for the highest input power (see Fig. 2). Almost the same break-up is experimentally observed in a comparable slab waveguide (unetched sample). The physics behind this effect can be interpreted in terms of the continuous limit, where this splitting is well known [11]. Above a certain threshold a two-soliton state is formed. Due to its lack of binding energy it finally splits in the presence of nonlinear absorption.

Although the action of any absorption is usually not appreciated, the soliton break-up generates many new and interesting physics, as we will demonstrate later. 


\subsection{Experiments with tilted beams}

The experiments on dynamical effects were devoted to externally induced lateral motions of discrete soliton. A glass wedge was positioned before the input lens in order to generate a tunable phase tilt across the beam. In this way, it was possible to vary the angle of propagation with respect to the input facet between 0 and about 1 degree (angle measured inside the waveguide).
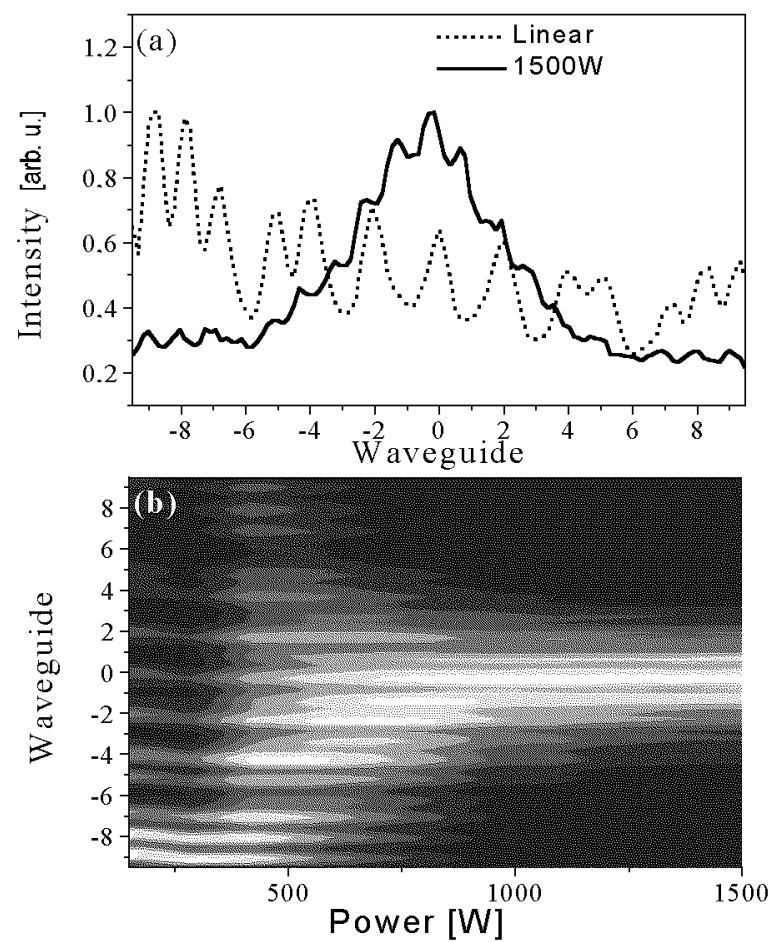

Fig. 3. Power induced locking of a discrete soliton for a small initial tilt: (a) field profiles for low (dashed line) and high (full line) power; (b) contour plot of the output field profiles vs. input peak power (initial tilt: $\approx 0.15^{\circ}$ in AlGaAs, beam size $9 \mu \mathrm{m}$ ).

In Figs. 3 and 4 we show the output power distribution of a narrow input beam $(9 \mu \mathrm{m}$ wide) travelling along the array for two different values of propagation angles. For low energy (see top of Figs. 3 and 4 ) the tilt results in a power redistribution in the array, but does not affect the basic structure of discrete diffraction. Most of the input energy is translated by about $80 \mu \mathrm{m}$ ( $8-9$ waveguides) when compared to the original input position. We note that this translation is not increased for bigger values of the input angle, but only the power imbalance between the two pronounced wings is increased (compare tops of Figs. 3 and 4). As stated above, once the geometry of the array is fixed, the final number of waveguides excited by the diffracting beam depends on the length of the sample rather than on the input angle. This is completely different from the continuous case where a 

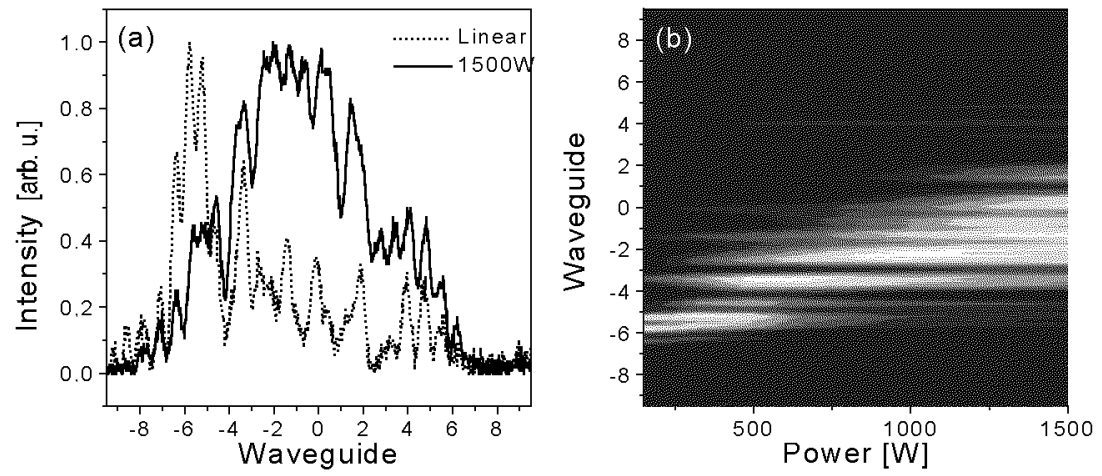

Fig. 4. Power induced locking of a discrete soliton for a large initial tilt: (a) field profiles for low (dashed line) and high (full line) power; (b) contour plot: output field profiles vs. input peak power (initial tilt $\approx 0.5^{\circ}$ in $\mathrm{AlGaAs}$, beam size $9 \mu \mathrm{m}$ ).

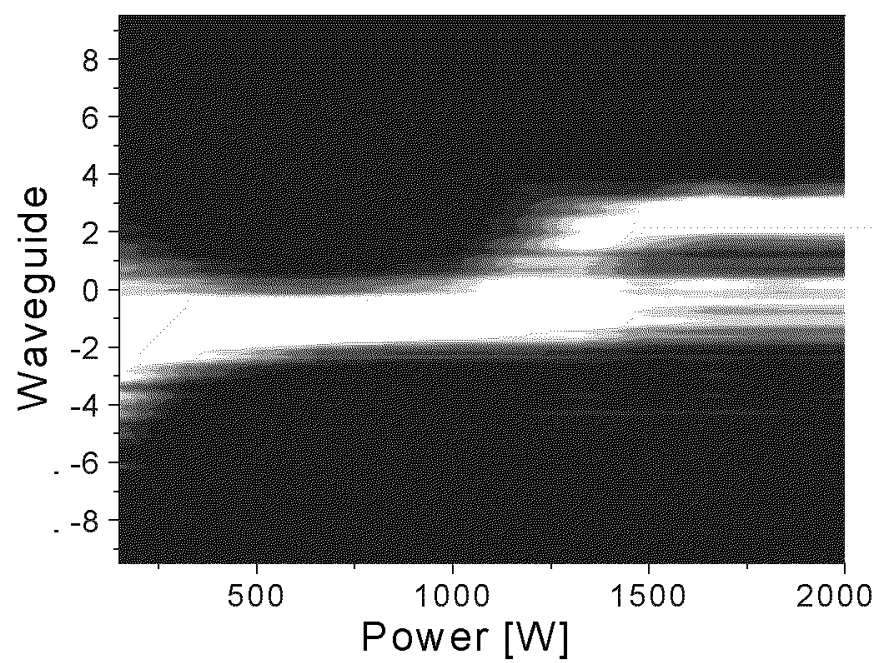

Fig. 5. "Antilocking" for a wide tilted beam, field profile vs. incoupled peak power (beam size $20 \mu \mathrm{m}$, input guide 0 , initial tilt $\approx 0.2^{\circ}$ in GaAs towards guides with $n<0$ ).

constant motion of the beam center with increasing tilt is observed. At least for small initial tilts the energy redistribution in the array is even faster than in a comparable slab waveguide. In case of Fig. 3 the same tilted beam injected into a comparable film waveguide of the same length would only experience a displacement of about $14 \mu \mathrm{m}$, where we observe a more than 5 times bigger shift in the array.

In the nonlinear case, the effects of discreteness are even more pronounced. Let us consider first the case of lower input tilt (see Fig. 3). As we increase the power we observe how the light distribution, initially shifted away from the input waveguide relocates back to the central position. This happens almost immediately as the discrete soliton is formed. However, the success of this process will depend 
critically from the initial input angle. For an increased tilt already in the low power case most of the power is confined to one of the diffraction wings. For increased input power the soliton forms but keeps on moving. For this large angle, we do not manage to lock the soliton in the central portion of the array even at the highest input power. Instead, we observe a continuous scan of the output position with increasing incident power.

The locking behavior described above was studied in literature [3] based on the DNLS. It demonstrates the power-induced transition from a quasicontinuous state with high mobility to a highly localized and almost locked one. For low input power, the soliton can follow the initial tilt, where the field is almost decoupled from the rest of the array for the highest power levels. If the initial beam is wide enough to allow for beam break-up nonlinear absorption may introduce some new effects which partially contradict this simple picture. Again, the soliton follows the tilt of the input beam for low input power levels first (see Fig. 5). For increased power levels, it starts to lock to the initial guide, but then it suddenly jumps

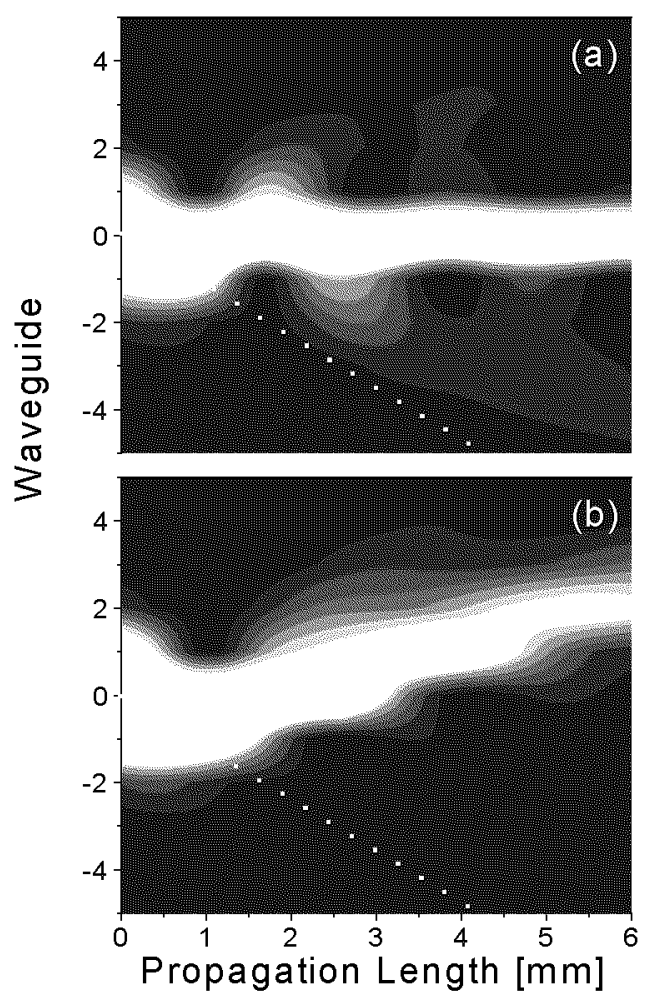

Fig. 6. cw-simulation of the field propagation for beam parameters similar as in Fig. 5 (peak power $1800 \mathrm{~W}$, dashed line: propagation direction of a low power beam): (a) simulations based on the DNLS (see Eq. (1)). The beam is locked, but oscillations around the final state occur; (b) CW-simulations with losses included. The beam turns into the direction opposite to the tilt. 
into the opposite direction. This "antilocking" is related to the beam break-up introduced above, but can be understood on the basis of cw simulations, if we accept slightly different values for the scaling and the powers predicted.

We assumed an initial beam similar to the experiment shown in Fig. 5. The power was chosen high enough to ensure locking and to allow for beam break-up, if absorption was added. In Fig. 6a we solved the DNLS for ideal conditions (cw, no absorption), where the soliton stays confined in the initial waveguide. Nevertheless, a closer look reveals damped oscillations around the final position and small outbursts of radiation during each oscillation period. If linear and nonlinear absorption are added, the power of the soliton is reduced during propagation. Consequently, its ability to lock to the initial waveguide diminishes. Now the radiation observed previously is becoming stronger and stronger. Finally, almost the whole residual of the beam leaves the initial guide at the end of the first oscillation period (see Fig. 6b).

Varying the input angle one finds a continuous transition from beam break-up, where the final power distribution is symmetric to the "antilocked" state, where almost all the power is contained in one of the beams leaving the initial guide.

\section{Conclusions}

In conclusion, we have experimentally demonstrated that discrete systems present dynamical properties, which differ considerably from those of continuous ones. We showed how, under certain conditions, an initially moving soliton can be captured at the initial waveguide, if a certain power is reached. If the power is high enough to induce beam break-up, an "antilocking" may occur. Hence, the soliton moves into a direction opposite to the initial tilt.

The author would like to acknowledge the Israeli Ministry of Science and the British Engineering Physical Science Research Council and the Deutsche Forschungsgemeinschaft (German Research Foundation) for financial support to this work.

\section{References}

[1] J.S. Aitchison, Y. Silberberg, A.M. Weiner, D.E. Leaird, M.K. Oliver, J.L. Jackel, E.M. Vogel, P.W.E. Smith, J. Opt. Soc. Am. B 8, 1290 (1990).

[2] D.N. Christodoulides, R.I. Joseph, Opt. Lett. 13, 794 (1988).

[3] A.B. Aceves, C. de Angelis, T. Peschel, R. Muschall, F. Lederer, S. Trillo, S. Wabnitz, Phys. Rev. E 53, 1172 (1996).

[4] H.S. Eisenberg, Y. Silberberg, R. Morandotti, A. Boyd, J.S. Aitchison, Phys. Rev. Lett. 81, 3383 (1998).

[5] A.S. Davydov, N.I. Kislukha, Phys. Status Solidi B 59, 465 (1995).

[6] P. Marquie, J.M. Bilbault, M. Remoissenet, Phys. Rev. E 51, 6127 (1995).

[7] E. Trías, J.J. Mazo, T.P. Orlando, Phys. Rev. Lett. 84, 741 (2000); P. Binder, D. Abraimov, A.V. Ustinov, S. Flach, Y. Zolotaryuk, Phys. Rev. Lett. 84, 745 (2000). 
[8] P. Denardo, B. Galvin, A. Greenfield, A. Larranza, S. Putterman, W. Wright, Phys. Rev. Lett. 68, 1730 (1992).

[9] T. Holstein, Ann. Phys. 8, 325 (1959).

[10] S. Flach, C.R. Willis, Phys. Rep. 295, 182 (1998).

[11] Y.V. Afanasjev, J.S. Aitchison, Y.S. Kivshar, Opt. Commun. 116, 331 (1995). 